\title{
Diagnostic nomogram model for predicting preoperative pathological grade of meningioma
}

\author{
Shijun Peng, Zhihua Cheng, Zhilin Guo \\ Department of Neurosurgery, The Ninth People's Hospital Affiliated to Shanghai Jiao Tong University Medical College, Shanghai, China \\ Contributions: (I) Conception and design: All authors; (II) Administrative support: Z Guo; (III) Provision of study materials or patients: Z Guo, \\ Z Cheng; (IV) Collection and assembly of data: S Peng; (V) Data analysis and interpretation: All authors; (VI) Manuscript writing: All authors; \\ (VII) Final approval of manuscript: All authors. \\ Correspondence to: Zhilin Guo, MD. Department of Neurosurgery, The Ninth People's Hospital Affiliated to Shanghai Jiao Tong University Medical \\ College, 639 Manufactory Road, Shanghai, 200001, China. Email: gzlysr@126.com.
}

\begin{abstract}
Background: Meningioma is the most common primary tumor of the central nervous system. Preoperative diagnosis of high-grade meningioma is helpful for the selection of treatment options. The aim of our study is to establish a diagnostic nomogram model for preoperative prediction of the pathological grade of meningioma.
\end{abstract}

Methods: The predictive model was established from a cohort of 215 clinicopathologically confirmed meningioma between January 2012 and December 2017. Radiomic features were collected from preoperative magnetic resonance imaging (MRI) and computed tomography of patients with meningioma. The least absolute shrinkage and selection operator (LASSO) regression model was used for data dimension reduction and feature selection. Multivariate logistic regression was used to build a predictive model and presented as a nomogram. The performance of the nomogram was assessed with respect to its calibration, discrimination, and clinical usefulness. Internal validation was evaluated using bootstrapping validation.

Results: High-grade meningioma was observed in 47 patients $(22 \%)$. The predictors included in the nomogram were tumor-brain interface, bone invasion, and tumor location. The final diagnostic model exhibited good calibration and discrimination with a C-index of 0.874 (95\% confidence interval: 0.818-0.929) and a higher C-index of 0.868 in internal validation. Decision curve analysis (DCA) indicated that the nomogram is very useful in clinical practice.

Conclusions: This study provides a nomogram model with tumor-brain interface, bone invasion, and tumor location that can effectively predict the preoperative pathological grading of patients with meningioma and thus help clinicians provide more reasonable treatment strategies for meningioma patients.

Keywords: Meningioma; nomogram; predictive model; grade; diagnosis

Submitted May 09, 2021. Accepted for publication Jul 16, 2021.

doi: $10.21037 /$ tcr-21-798

View this article at: https://dx.doi.org/10.21037/tcr-21-798

\section{Introduction}

Meningiomas are mainly derived from arachnoid cells and are the most common primary intracranial tumors, accounting for approximately $36.1 \%$ of all intracranial tumors (1-3). According to the World Health Organization (WHO) classification in 2016 (4), meningiomas are classified as grade I, II or III, among which high-grade (grade II and III) meningiomas account for approximately $18 \%$ of all meningiomas and often show aggressive disease, are difficult to completely eliminate and exhibit a high recurrence rate (5-7). Tumors are typically treated by surgical removal. If high grade lesions are pathologically evaluated during surgery, further treatments such as 
radiotherapy, chemotherapy or molecular targeted therapy may be required (8). Thus, the preoperative differential diagnosis of high-grade tumors is helpful to provide the most appropriate treatment. However, preoperative biopsy is difficult to perform, especially for tumors located in the skull base, and imaging is widely used in the primary diagnosis of meningioma $(9,10)$. The high proliferative potential or aggressive biological behavior of meningioma is related to certain specific magnetic resonance imaging (MRI) features. These features include hyperostosis, bony destruction, dural tail sign, unclear demarcation from brain tissue, intratumoral cystic change, and peritumoral brain edema (11). A meningioma typically has multiple radiological features (12). Assessing all relevant clinical and radiological characteristics is critical to predicting the preoperative grading of meningioma.

Therefore, a diagnostic nomogram model with imaging features was developed and validated to predict preoperative pathological grade in meningioma patients. We hope that this model will improve the diagnosis of preoperative patients with high-grade meningioma to help clinicians develop more appropriate treatment options.

We present the following article in accordance with the TRIPOD reporting checklist (available at https://dx.doi. org/10.21037/tcr-21-798).

\section{Methods}

\section{Patient selection}

The study was conducted in accordance with the Declaration of Helsinki (as revised in 2013). The study was approved by the ethics committee of Shanghai Ninth People's Hospital, Shanghai Jiao Tong University School of Medicine (SH9H-2021-T218-1), and individual consent for this retrospective analysis was waived. We reviewed all clinical data for all patients with postoperative pathologic findings of meningioma between 2012 and 2017 in our hospital. All included patients had clear preoperative MRI and CT images, and the tumors were surgically removed. Patients with incomplete preoperative CT and MRI images, repeated surgery, and no clear histopathological grade were excluded.

\section{Data collection}

Preoperative MRI and CT images were evaluated for tumor size and location, as well as tumor calcification or necrosis, tumor-brain interface, dural tail, bone invasion, and peritumoral edema. Tumor size was defined as the largest diameter of the tumor at any sequence of images. According to the different attachment sites of the tumor base, patients were divided into a skull base group and a non-skull base group. The non-skull base group included the sagittal sinus, falx cerebri, parietal ventricle, frontal lobe, temporal lobe, parietal lobe and occipital lobe. The skull base group included the anterior fossa, middle fossa, tentorium cerebelli, pontocerebellar crus, saddle, and sphenoid ridge. When the peritumoral rim is clear and the shape is regular without obvious lobules, a clear cerebrospinal fluid cleft is evident at the peritumoral margin. It can be defined as a clear tumor-brain interface, otherwise it is an unclear tumor-brain interface. The hyperintensity near the tumor on the preoperative $\mathrm{T} 2$-weighted image is defined as positive peritumoral edema. Non-contrast CT was used to assess tumor calcification and bone invasion. The $\mathrm{T} 2$ hyperintense nonenhancement region within the tumor was considered tumor necrosis.

\section{Statistical analysis}

We use the least absolute shrinkage and selection operator (LASSO) method to filter variables, which can prevent regression analysis from overfitting by complexity adjustment to make it more consistent with the actual situation. Then, the variables screened by LASSO regression were incorporated into the multivariate logistic regression analysis to construct the prediction model and are presented as a nomogram. The nomogram is based on the influence weight of each factor and provides a reasonable scoring threshold, and its predictive performance was measured by subject receiver operating characteristic (ROC) analysis using area under the curve (AUC) values. In addition, the clinical value of the nomograms was evaluated by calibration curve and decision curve analysis (DCA). Internal validation of the model was performed with the C-index calculated by bootstrapping (1,000 bootstrap resamples). All statistical tests and analyses were performed in $\mathrm{R}$ software version 4.0.3. Statistical significance was set at $<0.05$.

\section{Results}

\section{Patient demographics}

The basic characteristics of the study population are shown in Table 1. There were 168 (78\%) grade I patients and 47 
Table 1 Characteristics of patients

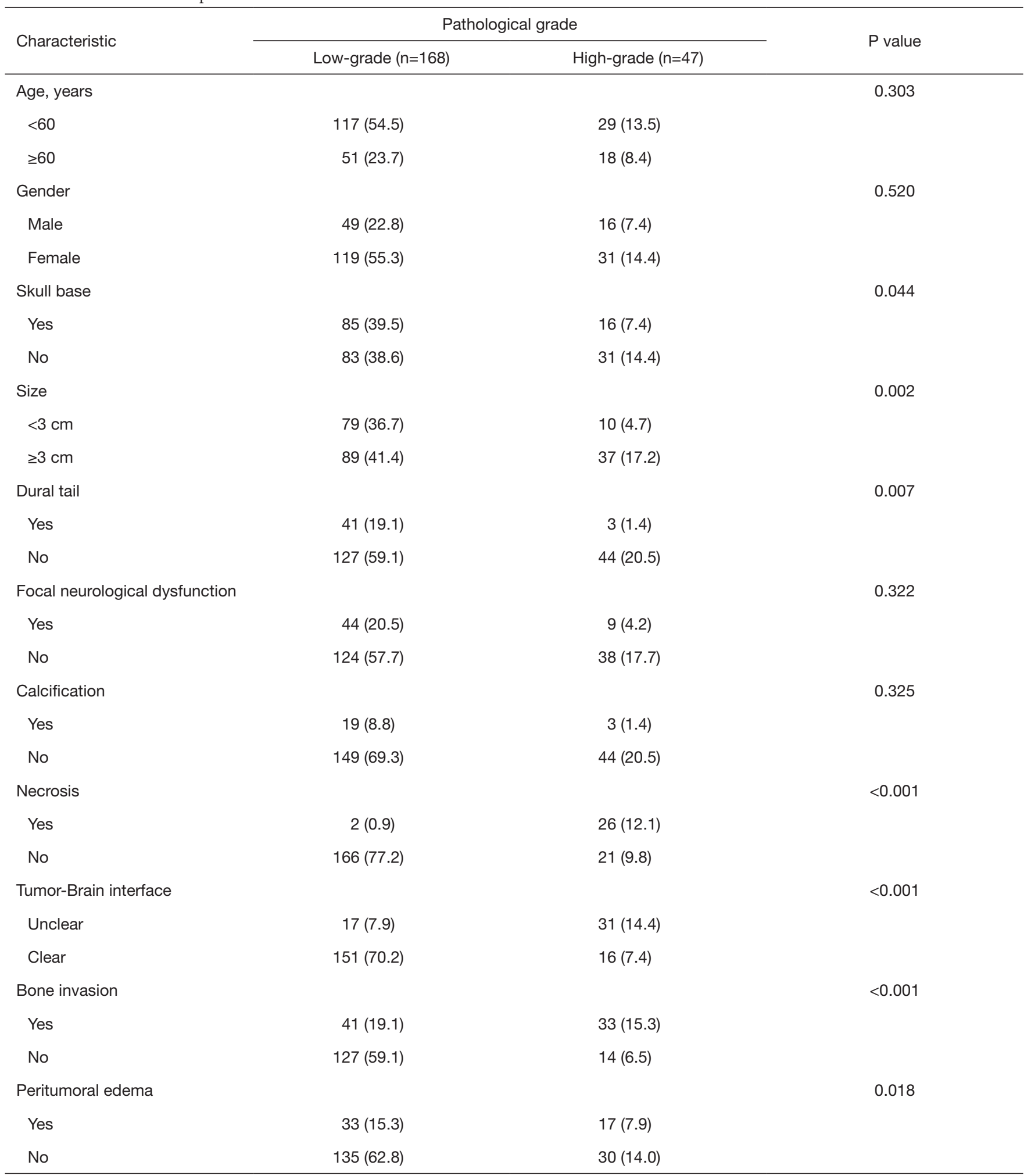

P-value is derived from the univariable association analyses between each of the characteristics and pathological grade of meningioma. 

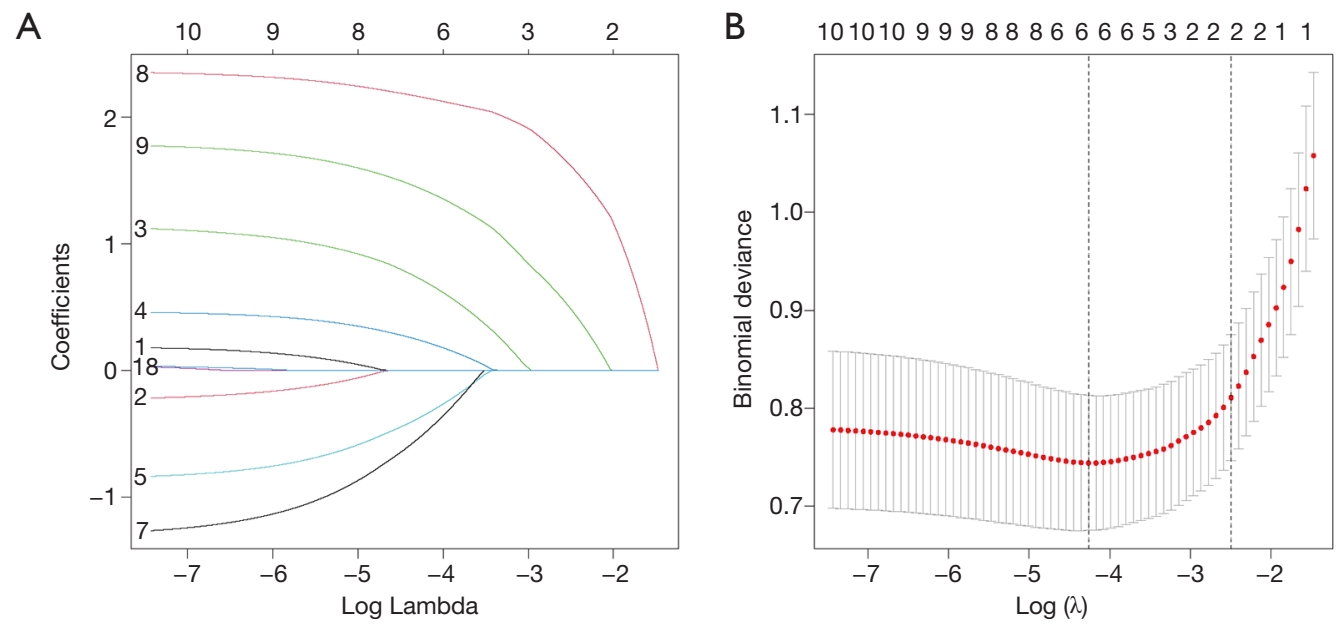

Figure 1 Feature's selection using the least absolute shrinkage and selection operator (LASSO). (A) A coefficient profile plot was produced against the $\log (\lambda)$ sequence. vertical line was drawn at the value selected chosen by 10 -fold cross-validation. (B) Tuning parameter ( $\lambda$ ) selection in the LASSO model used a 10-fold cross-validation via minimum criteria. The dotted vertical lines were drawn at the optimal values by using the minimum criteria and the 1 standard error of the minimum criteria (the 1-SE criteria).

(22\%) grade II and III patients. The mean age was 54 years (range, 10-83 years), and females accounted for the majority $(69.8 \%)$ with a high-level grade accounting for $14.4 \%$. ROC curves were used to determine the cutoff of size. Tumor size $\geq 3 \mathrm{~cm}$ could predict high-grade meningioma with a sensitivity of $79 \%$ and specificity of $47 \%$ ( $\mathrm{P}=0.007$ ). Tumor necrosis was highly heterogeneous in distribution with a specificity of $98 \%$ (OR, 102.762; 95\% CI, 22.743464.320; $\mathrm{P}<0.0001)$ and was not included in the model.

\section{Variable selection}

We used LASSO regression to select the possible predictors (Figure 1). Six features were identified as potential predictors in our study cohort. These features included tumor location, size, dural tail, calcification, tumor-brain interface, and bone invasion.

\section{Model construction}

Six variables determined by LASSO regression were included in the multivariate logistic analysis. Among these, tumor-brain interface, bone invasion and location were independent predictors of patients with high-grade meningioma. The above independent predictors were incorporated into the model and presented as a nomogram (Figure 2).

\section{Performance of the nomogram}

The C-index of the diagnostic prediction model was 0.874 (95\% CI, 0.818-0.929) in our study cohort, and 0.868 was verified internally by bootstrapping, indicating that the diagnostic model has good discrimination compared with single tumor-brain interface $(0.779)$, bone invasion $(0.729)$ and tumor location (0.583) models. In addition, the calibration curve of the nomogram for preoperative prediction of high-grade in patients with meningioma showed good agreement (Figure 3). Hosmer-Lemeshow test results were not statistically significant $(\mathrm{P}=0.378)$. These results indicate that no significant difference was noted between the prediction of the model and the actual observation results. The high degree of discrimination and good consistency indicate that this prediction model exhibits good reliability for the prediction of actual results.

\section{Clinical use}

The DCA for the diagnostic nomogram and each single predictor model is shown in Figure 4. The decision curve indicates the net benefit of patients when intervention is performed under various threshold probabilities. When the prediction model threshold probability is $29 \%$ of the best diagnosis probability, the diagnostic nomogram model has the highest net benefit followed by a single model of tumor- 


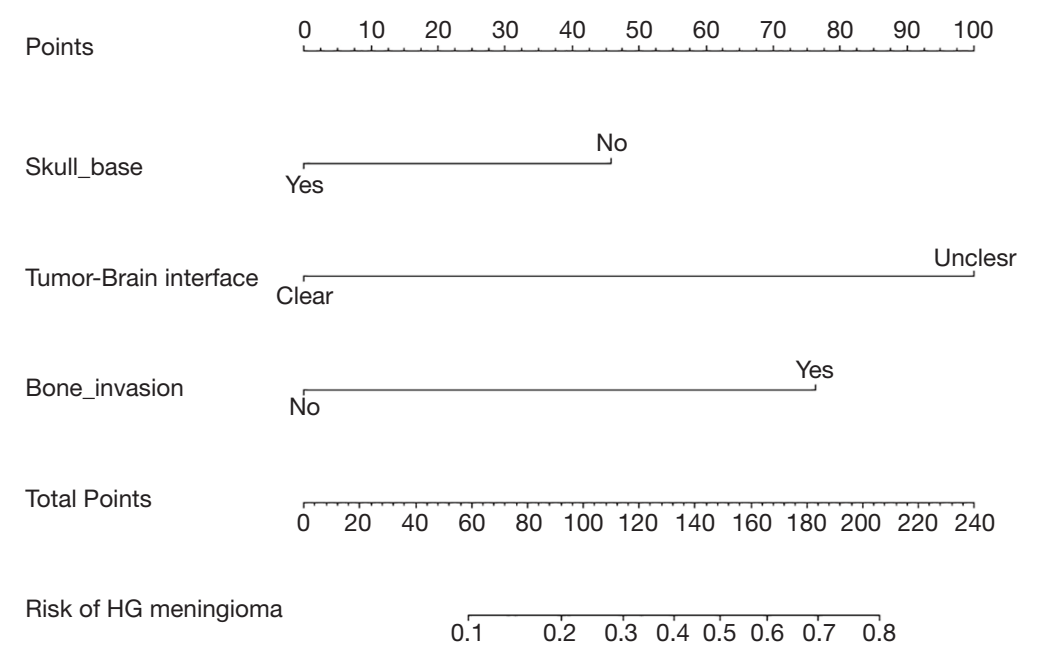

Figure 2 Diagnostic nomogram. The nomogram was constructed from tumor-brain interface, bone invasion, and location of the tumor. HG, high-grade.

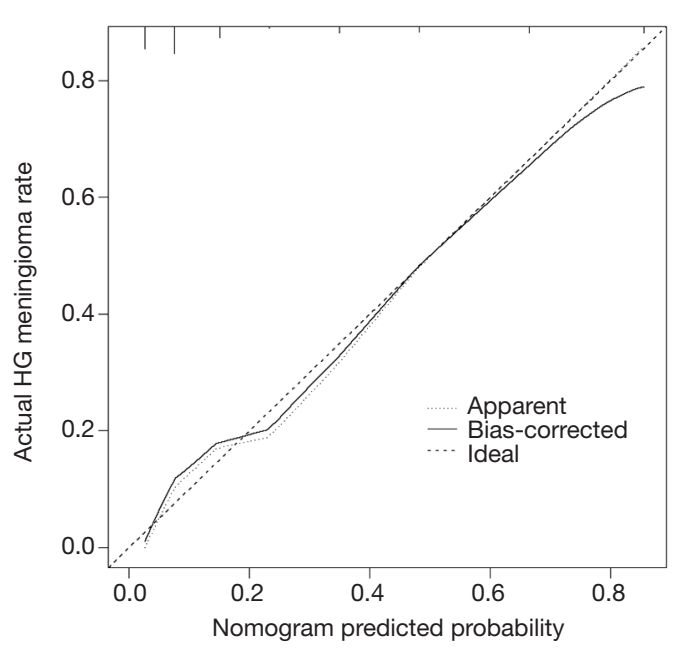

Figure 3 Calibration plot. The prediction results were consistent with the diagonal line, which indicates that the prediction results are accurate.

brain interface.

\section{Discussion}

The nomogram was used to predict the risk of meningioma recurrence (13). Nassiri et al. constructed a nomogram to well predict the risk of early recurrence by combining DNA methylation molecular factors and determined prognostic clinical factors. Also, the pathological grading

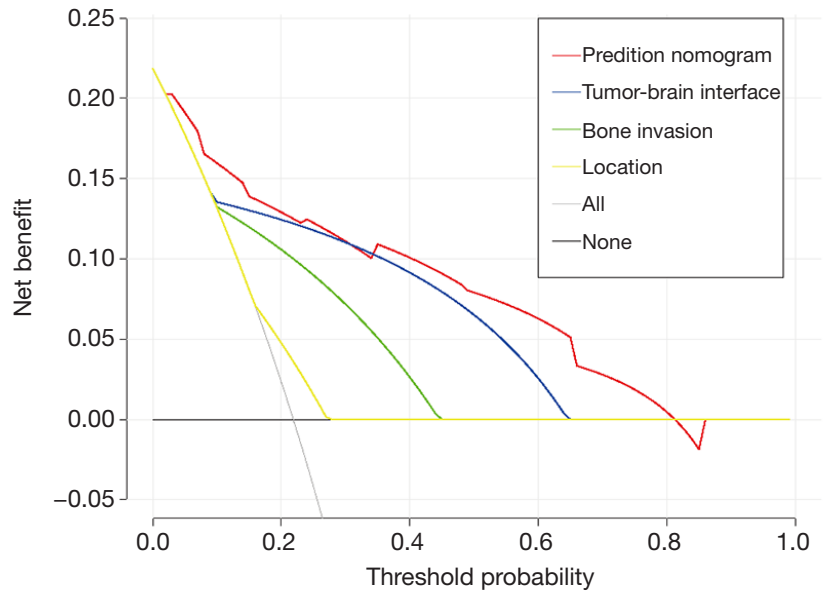

Figure 4 Decision curve analysis for the diagnostic nomogram. The net benefit was calculated by subtracting the proportion of all false positive patients from the proportion of true positives, and was then weighted based on the associated harms of prior treatment and outcomes that did not require treatment.

of meningioma can be predicted by constructing a preoperative model based on relevant characteristics. However, compared to combining DNA methylation molecular factors, the pathological grading factors of meningioma are obvious in radiological features, and the model constructed by multiple imaging features is relatively simple and practical in clinical practice. In addition, the correlation between the imaging features of different pathological grades can also be analyzed to discuss the 
radiological features of high-grade meningioma. We developed and validated a diagnostic prediction tool for the preoperative individualized prediction of the pathological grade of meningioma. A predictive model was constructed by including preoperative predictive factors and represented in a nomogram. Most of the useful markers were selected using LASSO regression to reduce the regression coefficient to test the correlation of the predicted results. This method not only selects predictors according to the bias of their univariate correlation with clinical outcomes but also combines the selective predictors into a comprehensive model. The nomogram consists of multiple predictors and has good calibration and discrimination in our cohort (C-index, 0.874), especially our high C-index (C-index, 0.868 ) in the interval validation. Nevertheless, it still requires a larger sample size for external verification of its reliability. Through the DCA curve, the net benefit of the patient under the predictive model's diagnostic probability can be obtained, which may help clinicians to determine the appropriate threshold probability for treatment.

When tumor growth invades the surrounding brain tissue, it appears to be unclear in the brain tissue on MRI, especially in atypical meningioma and malignant meningioma (4). Therefore, the pathological type of the tumor can be judged by the tumor-brain interface of the tumor on the preoperative MRI image to develop an appropriate surgical plan. In recent univariate and multivariate analyses, the unclear boundary between tumor and brain tissue was an important predictor of high-grade meningioma $(12,14)$. In this study, $65 \%$ of patients with unclear tumor-brain interface had postoperative pathology of high-grade meningioma, with a sensitivity of $66 \%$ and a specificity of $90 \%$. Although the tumor-brain interface can be used as an independent predictor $(\mathrm{P}<0.001)$, the prediction model of tumor-brain interface combined with other preoperative factors exhibits a higher diagnostic ability for high-grade meningioma (AUC=0.874) compared with the single model (AUC=0.779). In addition, $70 \%$ of highgrade meningioma present with bone destruction before surgery. Bone invasion is an independent predictor of highgrade meningioma $(\mathrm{P}<0.001)$. This does not mean that a correlation exists between tumors located at the skull base and high-grade meningioma. In contrast, previous studies have found that non-skull base tumors are important independent risk factors for high-grade meninges (12,15-20). which is consistent with our findings (OR 3.042, 95\% CI, $1.223-7.567, \mathrm{P}=0.017$ ).

In our study, tumor size was significantly different in univariate analysis. Some studies have also suggested that tumor size can predict histopathological tumor grade and is a strong predictor of atypical meningioma pathology $(21,22)$. Even after controlling for the interaction of sex and other risk factors for meningioma, tumor size is also related to grade II meningioma (23). Judging from the basic characteristics of tumors, grade II and III meningioma grow faster than benign tumors, but this may also be due to the gradual enlargement of tumors that become more aggressive. Therefore, the size of a single tumor cannot be a distinguishing feature of high-grade meningioma. Under the influence of multiple variables, our multivariate logistic regression analysis indicates that tumor size $\geq 3 \mathrm{~cm}$ cannot be an independent predictor of high-grade meningioma. Of course, a smaller sample size and inappropriate tumor size classification will cause errors. Therefore, a larger sample size and specific research on tumor growth mechanisms can help clarify the relationship between tumor size and highgrade meningioma.

Whether a correlation exists between peritumor edema and meningioma grade is controversial. The etiology of peritumor edema remains unclear, and peritumor venous congestion or ischemia has become the mainstreamview (24). Some studies have demonstrated that peritumor edema is an important feature of atypical meningioma or malignant meningioma, whereas other studies have also noted that a relationship exists between peritumor edema and meningioma grade. However, its statistical significance has not been demonstrated in previous studies $(14,25)$. The subjectivity of the diagnosis of peritumoral edema and other influential factors, such as tumor size, may interfere with the results. In this study, peritumoral edema was significantly correlated with tumor size $(\mathrm{P}=0.001)$. In univariate analysis, positive peritumoral edema was a predictor of meningioma grade (OR 2.318, 95\% CI, 1.144-4.698, $\mathrm{P}=0.02$ ), but peritumoral edema was not an independent predictor of highgrade meningioma based on multivariate logistic regression analysis. Furthermore, our study did not explore the correlation between the degree of edema and meningioma grade. Studies have shown that moderate or severe edema is more common in grade II and III meningioma (26), so it is necessary to study the correlation between the degree of peritumoral edema and high-grade meningioma in an expanded sample size model.

Our current study also has some limitations. First, the nomogram model for diagnostic prediction was established with a small sample size, and the included predictors could not include all the potential factors with diagnostic efficacy. 
Although the accuracy of our nomogram model performs well through internal validation of bootstrap testing, external validation of multiple agencies can provide more convincing evidence. In addition, the study is a singlecenter retrospective study with inherent defects. Prospective studies in a larger population are needed to further validate the results obtained.

\section{Conclusions}

This study built a prediction model for preoperative meningioma grade, and the model of predicting factors, including clear tumor-brain interface, bone invasion and non-skull base locations, exhibited a better prediction effect. We believe that the establishment of the model can be effective for preoperative diagnosis of high-grade meningioma, which is more suitable for patients with meningioma treatment. Further external validation can make the model more accurate.

\section{Acknowledgments}

Funding: None.

\section{Footnote}

Reporting Checklist: The authors have completed the TRIPOD reporting checklist. Available at https://dx.doi. org/10.21037/tcr-21-798

Data Sharing Statement: Available at https://dx.doi. org/10.21037/tcr-21-798

Peer Review File: Available at https://dx.doi.org/10.21037/ tcr-21-798

Conflicts of Interest: All authors have completed the ICMJE uniform disclosure form (available at https://dx.doi. org/10.21037/tcr-21-798). The authors have no conflicts of interest to declare.

Ethical Statement: The authors are accountable for all aspects of the work in ensuring that questions related to the accuracy or integrity of any part of the work are appropriately investigated and resolved. The study was conducted in accordance with the Declaration of Helsinki (as revised in 2013). The study was approved by the ethics committee of Shanghai Ninth People's Hospital,
Shanghai Jiao Tong University School of Medicine (SH9H2021-T218-1), and individual consent for this retrospective analysis was waived.

Open Access Statement: This is an Open Access article distributed in accordance with the Creative Commons Attribution-NonCommercial-NoDerivs 4.0 International License (CC BY-NC-ND 4.0), which permits the noncommercial replication and distribution of the article with the strict proviso that no changes or edits are made and the original work is properly cited (including links to both the formal publication through the relevant DOI and the license). See: https://creativecommons.org/licenses/by-nc-nd/4.0/.

\section{References}

1. Park YW, Oh J, You SC, et al. Radiomics and machine learning may accurately predict the grade and histological subtype in meningiomas using conventional and diffusion tensor imaging. Eur Radiol 2019;29:4068-76.

2. Nowosielski M, Galldiks N, Iglseder S, et al. Diagnostic challenges in meningioma. Neuro Oncol 2017;19:1588-98.

3. Ostrom QT, Gittleman H, Liao P, et al. CBTRUS Statistical Report: Primary brain and other central nervous system tumors diagnosed in the United States in 20102014. Neuro Oncol 2017;19:v1-v88.

4. Louis DN, Perry A, Reifenberger G, et al. The 2016 World Health Organization Classification of Tumors of the Central Nervous System: a summary. Acta Neuropathol 2016;131:803-20.

5. Buerki RA, Horbinski CM, Kruser T, et al. An overview of meningiomas. Future Oncol 2018;14:2161-77.

6. Ros-Sanjuan A, Iglesias-Moroño S, Carrasco-Brenes A, et al. Atypical Meningiomas: Histologic and Clinical Factors Associated With Recurrence. World Neurosurg 2019;125:e248-56.

7. Aizer AA, Bi WL, Kandola MS, et al. Extent of resection and overall survival for patients with atypical and malignant meningioma. Cancer 2015;121:4376-81.

8. Apra C, Peyre M, Kalamarides M. Current treatment options for meningioma. Expert Rev Neurother 2018;18:241-9.

9. Goldbrunner R, Minniti G, Preusser M, et al. EANO guidelines for the diagnosis and treatment of meningiomas. Lancet Oncol 2016;17:e383-91.

10. Huang RY, Bi WL, Griffith B, et al. Imaging and diagnostic advances for intracranial meningiomas. Neuro Oncol 2019;21:i44-61. 
11. Hanft S, Canoll P, Bruce JN. A review of malignant meningiomas: diagnosis, characteristics, and treatment. J Neurooncol 2010;99:433-43.

12. Lin BJ, Chou KN, Kao HW, et al. Correlation between magnetic resonance imaging grading and pathological grading in meningioma. J Neurosurg 2014;121:1201-8.

13. Nassiri F, Mamatjan Y, Suppiah S, et al. DNA methylation profiling to predict recurrence risk in meningioma: development and validation of a nomogram to optimize clinical management. Neuro Oncol 2019;21:901-10.

14. Salah F, Tabbarah A, ALArab YN, et al. Can CT and MRI features differentiate benign from malignant meningiomas? Clin Radiol 2019;74:898.e15-23.

15. Czyz M, Radwan H, Li JY, et al. Fractal Analysis May Improve the Preoperative Identification of Atypical Meningiomas. Neurosurgery 2017;80:300-8.

16. Hwang WL, Marciscano AE, Niemierko A, et al. Imaging and extent of surgical resection predict risk of meningioma recurrence better than WHO histopathological grade. Neuro Oncol 2016;18:863-72.

17. Kasuya H, Kubo O, Tanaka M, et al. Clinical and radiological features related to the growth potential of meningioma. Neurosurg Rev 2006;29:293-6; discussion 296-7.

18. Cornelius JF, Slotty PJ, Steiger HJ, et al. Malignant potential of skull base versus non-skull base meningiomas: clinical series of 1,663 cases. Acta Neurochir (Wien) 2013;155:407-13.

Cite this article as: Peng S, Cheng Z, Guo Z. Diagnostic nomogram model for predicting preoperative pathological grade of meningioma. Transl Cancer Res 2021;10(9):4057-4064. doi: $10.21037 /$ tcr-21-798
19. Kane AJ, Sughrue ME, Rutkowski MJ, et al. Anatomic location is a risk factor for atypical and malignant meningiomas. Cancer 2011;117:1272-8.

20. Sade B, Chahlavi A, Krishnaney A, et al. World Health Organization Grades II and III meningiomas are rare in the cranial base and spine. Neurosurgery 2007;61:1194-8; discussion 1198.

21. Magill ST, Chae R, Aghi MK, et al. Larger meningioma size at presentation is associated with higher tumor grade. J Neurol Surg B Skull Base 2017;78:S1-156.

22. Hale AT, Wang L, Strother MK, et al. Differentiating meningioma grade by imaging features on magnetic resonance imaging. J Clin Neurosci 2018;48:71-5.

23. Magill ST, Young JS, Chae R, et al. Relationship between tumor location, size, and WHO grade in meningioma. Neurosurg Focus 2018;44:E4.

24. Yin T, Zhang J, Zhang H, et al. Poor Brain-Tumor Interface-Related Edema Generation and Cerebral Venous Decompensation in Parasagittal Meningiomas. World Neurosurg 2018;115:e544-51.

25. Lu Y, Liu L, Luan S, et al. The diagnostic value of texture analysis in predicting WHO grades of meningiomas based on ADC maps: an attempt using decision tree and decision forest. Eur Radiol 2019;29:1318-28.

26. Zhang T, Yu JM, Wang YQ, et al. WHO grade I meningioma subtypes: MRI features and pathological analysis. Life Sci 2018;213:50-6. 